\title{
Aportes de los Programas Universitarios para Mayores al Valor Social de la Educación a lo largo de la vida. Reflexiones a partir del estudio de un caso
}

\author{
Contributions of University Programs for Seniors to the Social \\ Value of Education throughout life. Reflections from the study of a \\ case
}

\author{
Judith Quintano Nieto \\ Sonia Ortega Gaite \\ María Tejedor Mardomingo \\ Universidad de Valladolid
}

Recibido: $12 / 10 / 2018$

Aceptado: $14 / 12 / 2018$

\begin{abstract}
Education is a constant in every human being's life; throughout the life cycle, many and different learnings are generated, which must have an impact on the educational and social institutions by means of educational itineraries throughout life in any space and time. The work presented here aims to draw attention to the importance of those adult education modalities that are not part of the regulated educational system, but are part of lifelong learning. It specifically focuses on the University Programs for Seniors, and it particularly explores the personal perception of the Social Value of Education (VSE-Subjective) of the participants in this type of programs, through their evaluations reflected in the questionnaires and interviews of the students Guardo, a town in the north of Palencia. This analysis reveals the educational value that is concealed in these experiences, shown in three levels: cultural, personal and social. In addition, it proves the need for these university programs that are highly valued by their participants and which are a sign of the education and culture strength as a tool for personal improvement and social and civic transformation in the key of social justice.
\end{abstract}

KEY WORDS: lifelong learnig; university extension; adult education; social value of education; community education

\section{RESUMEN}

La educación es un constante en la vida del ser humano; a lo largo del ciclo vital se gestan numerosos y diversos aprendizajes que deben tener repercusión en las instituciones educativas y sociales a través de itinerarios educativos a lo largo de la vida en cualquier espacio y tiempo. El trabajo que aquí se presenta pretende visibilizar la importancia de aquellas modalidades de educación de personas adultas que no se enmarcan en el sistema educativo reglado, sino que forman parte del aprendizaje a lo largo de la vida. Específicamente se centra en los Programas Universitarios para Mayores, y más concretamente se indaga en las percepciones personales del Valor Social de la Educación (VSESubjetivo) de los participantes en este tipo de programas, a través de sus valoraciones reflejadas en cuestionarios y entrevistas de alumnado representativo de Guardo, localidad del norte de Palencia. De este análisis se desprende el valor educativo que subyace a estas experiencias en tres niveles: cultural, personal y social. Además, se justifica la necesidad de estos programas universitarios, enormemente valorados por sus participantes y que son una muestra de la fuerza de la educación y la cultura como herramienta de mejora personal y transformación social y ciudadana en clave de justicia social.

PALABRAS CLAVE: aprendizaje a lo largo de la vida; extensión universitaria; educación de adultos; educación social; valor social de la educación 


\section{Introducción}

Resulta incuestionable el Valor Social de la Educación a lo largo de la vida, su necesidad en las distintas situaciones y circunstancias por las que atraviesa el recorrido vital del ser humano. "La Educación probablemente es el mejor instrumento de las políticas sociales para orientar la transformación y la mejora de las personas, los colectivos y las sociedades" (Jornet, 2012, p.354). La educación permanente y a lo largo de la vida están vinculadas muy estrechamente a la educación social, pues tienen entre sus manos utilizar la educación como factor de transformación y cohesión social potenciando así una vida democrática. (Quintano, 2014).

El dibujo poblacional presente y futuro denota el incremento de personas mayores de sesenta años en nuestras sociedades. El progresivo envejecimiento hace patente la necesidad de espacios educativos para personas adultas y mayores, como son los Programas Universitarios para Mayores (PUM) que se desarrollan en muchas de las instituciones universitarias.

Los PUM, en constante crecimiento por toda la geografía española, desde su creación han jugado un papel importante como herramienta eficaz y favorable para el envejecimiento activo, partícipe y protagonista del desarrollo social, cultural y científico de la sociedad en la que convive (Pozón, 2018).

La importante función social que desempeñan estos programas, además de beneficiar a la población adulta-mayor, también incide en las instituciones universitarias pues gracias a ellos ven proyectada su acción cultural, científica e investigadora en otros sectores de la sociedad, lo que supone una apertura socializadora de las mismas contribuyendo a universalizar la cultura que convierte a las universidades en espacios más abiertos y más cercanos a la sociedad en general (Fernández-García, García \& Pérez, 2014).

\section{El aprendizaje a lo largo de la vida y sus aportes al Valor Social de la Educación (VSE)}

$\mathrm{El}$ aprendizaje es una constante en la vida de toda persona, aprendemos desde que nacemos hasta el fin de nuestros días. En tiempos de crisis, personas, instituciones y sociedades deben aunar fuerzas y reivindicar más cultura, más aprendizaje, más conocimiento, porque es un derecho y la llave que abrirá las puertas a la sociedad global del conocimiento, supondrá mejor calidad de vida y también reforzará las capacidades personales y sociales.

La educación confía en las personas y considera que todas tienen un elevado potencial de aprendizaje que ha de ser desarrollado para que puedan sentirse protagonistas de sus propias vidas, tomen las decisiones de manera autónoma y participen activamente en la realidad de constante transformación a la que pertenecen.

La sociedad se está enfrentando a un cambio estructural en casi la totalidad de los ámbitos que la conforman, lo que está provocando una irreversible evolución en profundidad de la sociedad global. Por ello, es imprescindible la educación en todas las etapas de la vida del ser humano, abarcando el desarrollo personal y social de todo tipo y en cualquier escenario (OCDE,1996, citado en Morón, 2014, p.112).

El aprendizaje a lo largo de la vida y la educación de personas adultas se reconoce en numerosos discursos, políticos y pedagógicos, como instrumento clave para afrontar los retos sociales y del desarrollo en todo el mundo. No obstante, en numerosas ocasiones, no se traslada a la correspondiente integración, priorización política y asignación de recursos.

Las agencias educativas y sociales han de asumir que este tipo de educación debe estar, ligada a los nuevos agentes, escenarios y formas de aprendizaje, pues los cambios que acontecen demandan una permanente actualización de los aprendizajes. El mayor acceso a la cultura, la escolaridad universal y el aumento de la esperanza de vida generan nuevas necesidades de aprendizaje en el plano personal y profesional, que sumado a los cambios hacia una sociedad más participativa, multicultural y sostenible requieren que se mejoren las competencias sociales para que la ciudadanía pueda desenvolverse en una sociedad más compleja.

El modelo poblacional actual y el crecimiento progresivo que se prevé exigen que se garantice el derecho a la educación de las personas mayores atendiendo a las premisas mencionadas, 
repensando modelos que estimulen la autoestima y la realización personal y colectiva, que den respuesta a las nuevas situaciones sociales, políticas, económicas y culturales que nos plantea el mundo globalizado.

En conclusión, es posible y conveniente una construcción más social del aprendizaje a lo largo de la vida en clave de educación ciudadana y fundamentada en los valores de igualdad e inclusión (Vargas, 2017).

La literatura históricamente nos plantea tres modalidades de educación diferentes: formal, no formal e informal. Esta tipificación, que abarca a la educación en general, se ha extendido desde los años 70 (Coombs, 1971), ha ido proliferando y resulta de uso común en el lenguaje pedagógico incluyendo también a la educación permanente (Cabello, 2002).

Desde una concepción más holística de la educación y de la pedagogía, son ya numerosos los autores que enfatizan la necesidad de reconceptualización de esos términos, sobre todo haciendo alusión a la formalidad y valor de todas las educaciones posibles que emanan de muy diversas agencias y tiempos (Ortega, 1998; Flecha \& Elboj, 2000; Billet, 2002; Caride, 2004; Ortega, 2005; UNESCO, 2005; Núñez, 2010; Martí \& Gil, 2012; UNESCO, 2013).

"El reconocimiento de la educación a lo largo de toda la vida desvela que la formación se extiende más allá de los límites infantoescolares, descubriendo un aspecto formativo que ya existía, la educación de personas adultas" (Moreno-Crespo, 2015, p.116). La educación escolar es imprescindible para el desarrollo de las personas y, sin duda alguna, será uno de los instrumentos que más favorezcan oportunidades para la inclusión y ejercicio de la ciudadanía. No obstante, el valor de la educación social es también indispensable, pues este tipo de educación apuesta por una educación continua que incluya todos aquellos aprendizajes que contribuyen a la mejora de la calidad de vida de las personas a través de la transformación autónoma de su entorno, así como, aquellos que favorecen las relaciones interpersonales y de convivencia social, por lo que se tienen en cuenta una gran cantidad de entornos de los que se desprenden actos de aprendizaje.

Partimos de la definición del Valor Social de la Educación (VSE) aportada por Jornet, Perales y Sánchez-Delgado (2011) concebido como:

"la utilidad que tiene la educación dentro de una sociedad para el desarrollo y la promoción de las personas en los ámbitos social y laboral, así como a las ventajas que aporte como elemento de prevención de la exclusión social, y como garantía para el desarrollo y la mejora de su bienestar a lo largo de la vida". (p.52)

Desde una perspectiva objetiva del VSE se analiza la educación como un instrumento que aporta mejores niveles de desarrollo y promoción de las personas en los ámbitos social y laboral. Algunos de los indicadores propios de esta perspectiva hacen referencia a la valoración que la sociedad hace de la educación, que puede verse retratada en los esfuerzos, en relación con "las inversiones en educación" (Sancho-Álvarez, Jornet, \& González-Such, 2016, p.334), que se realizan para desarrollar acciones educativas a lo largo de la vida. Por otro lado, también, los efectos que tiene la educación en el bienestar personal y social, en términos de justicia social y movilidad social, entre otros.

En lo relacionado con la perspectiva subjetiva del VSE se hace referencia a las "percepciones personales y sociales de la población ante la importancia de la educación" (Sancho-Álvarez, Jornet, \& González-Such, 2016, p.335) valorando la importancia percibida de la misma para la promoción social, laboral y del bienestar personal y colectivo, a lo largo de la vida. De esas percepciones surgen imaginarios sociales vinculados a la satisfacción personal y la justicia social. La satisfacción personal que aporta la educación favorece la motivación personal generando altas responsabilidades ante la misma. Vinculado a ello está también la justicia social, pues a través de la formación planificada dentro de los proyectos educativos o de aquella invisible y no intencional que también entra en escena, en las acciones educativas se educa en valores que pueden generar trasformaciones en las relaciones entre personas en clave de paz, respeto e igualdad, y esto se hace a lo largo de toda la vida. 
Queda así justificada la necesidad y el valor social de la educación a lo largo de la vida. Por ello, la institución académica no es la única encargada de educar para dar respuesta a los desafíos personales y sociales que acontecen en el ciclo vital; todas las entidades educativas y en todas sus tipologías han de contemplarlo. Las distintas modalidades de educación han de concebirse "como partes de un todo, que solo adquieren su verdadero sentido y esencia en esa relación con el todo" (Cabalé \& Rodríguez, 2017, p.6).

El objeto del trabajo presentado, por tanto, es analizar el Programa Interuniversitario de la Experiencia de la sede rural de Guardo en clave de Valor Social de la Educación subjetivo, desde la dimensión de valor intrínseco diferencial de la educación. Es interesante valorar la cantidad de aspiraciones educativas que tienen las personas que participan, y la satisfacción vital que les aporta su propia experiencia como educandos, valorando la importancia que tiene este tipo de educación, ya no para lo relacionado con el ámbito laboral y empresarial, sino para el personal y colectivo, pues "si la población ve en la educación un factor clave de desarrollo personal y transformación, es obvio que incrementara su interés sobre la misma, en lo político y en lo personal" (Sancho-Álvarez, Jornet, \& González-Such, 2017, p.4).

\section{Los Programas Universitarios para Mayores}

El envejecimiento es una realidad demográfica que se hace especialmente patente en aquellos países con mayores tasas de desarrollo socioeconómico donde se consigue vivir hasta edades avanzadas que, en épocas anteriores, eran prácticamente impensables; un envejecimiento demográfico que está suponiendo uno de los mayores retos políticos sociales y culturales. Pero esta actual eclosión de la actividad formativa para las personas adultas mayores no se explica atendiendo sólo a razones de índole demográfica sino también de trascendencia pedagógica. El acceso a la cultura de las personas mayores del mundo urbano, y especialmente del mundo rural, supone su progresiva inclusión como ciudadanos y ciudadanas activos en la sociedad del conocimiento.

La progresiva adquisición de esta condición de ciudadano o ciudadana dentro de un marco de aprendizaje a lo largo de la vida exige apostar por una educación transformadora, igualitaria, continua y democrática, cuyos objetivos sean desarrollar la autonomía y el sentido de la responsabilidad de las personas y las comunidades, reforzar la capacidad de hacer frente a las transformaciones de la economía, la cultura y la sociedad en su conjunto y promover la coexistencia, la tolerancia y la participación consciente y creativa de la ciudadanía en su comunidad.

En esta misma línea, el informe Active Ageing: A Policy Framework (OMS, 2002, p.12) define Envejecimiento Activo como "el proceso de optimizar las oportunidades para la salud, participación y seguridad al objeto de mejorar la calidad de vida de las personas a medida que envejecen". Superando el enfoque de prevención de la enfermedad y cuidado de la salud introduce aspectos relacionados con los derechos de las personas mayores, el aprendizaje continuo y a lo largo de la vida, y la importancia de la participación social y ciudadana en los procesos de envejecimiento.

Los procesos educativos adultos suponen exponencialmente una enorme transcendencia social puesto que enriquecen nuestro mundo aportando perspectivas diversas que generan transformación social, compartiendo el conocimiento gestado durante muchos años de vida personal social y laboral. Se trata de generar nuevos contextos de participación y aprendizaje compartido a los que las universidades no pueden ser ajenas.

La Universidad cumple con su vocación de universalidad, que no es otra que la de contribuir al desarrollo de los hombres y mujeres que deciden aumentar su calidad de vida sin límite de edad, constituyéndose como un espacio en el que intercambiar experiencias, con la cultura y el conocimiento como telón de fondo. Esto se plantea en consonancia con la investigación y directrices políticas y administrativas actuales de envejecimiento activo pues nos indican que es un deseo ya logrado vivir más años, por lo que la meta perseguida ahora es dotarlos de calidad de vida.

Los PUM son una realidad en la mayoría de las universidades europeas y en muchas universidades de América Latina desde hace varias décadas. El término "Programas Universitarios para Adultos Mayores" incluye todos aquellos proyectos universitarios de índole educativa y cultural 
para personas con más de 50 años con una doble perspectiva, la extensión universitaria, y la difusión y transferencia de la ciencia y el conocimiento. Bajo este epígrafe, se incluyen experiencias de diversa índole, estructura y organización que pretenden acercar la universidad a la sociedad en toda su amplitud: conocimiento, espacios compartidos, servicios, investigación, etc.

Con estos programas se pretende crear situaciones óptimas en las que se genere el diálogo y surja el aprendizaje desde el debate y el intercambio de saberes. "Se parte de la hipótesis de que las personas mayores tienen una serie de conocimientos adquiridos a lo largo de la vida y que el profesorado se debe encargar de actualizar esos contenidos y de introducir otros nuevos a través del aprendizaje dialogado" (Tejedor \& Rodríguez, 2008, p.101).

Como se puede observar en la figura 1 el número de personas matriculadas en los PUM así como el número de universidades que los ofertan ha aumentado exponencialmente en los últimos años en España. Resulta palmario comprobar que cerca de 60.000 personas (incluyendo programas universitarios no agrupados en la Asociación Estatal de Programas para Universitarios MayoresAEPUM) acuden a diario a las universidades a formarse, a relacionarse socialmente, a actualizar conocimientos o a dotarlo de un mayor significado. Como se ha comprobado (Tejedor \& Rodríguez, 2008), si bien el origen de estos programas tenía un matiz compensatorio, permitir el acceso a la universidad a quienes no pudieron acudir en su momento, cada vez los PUM acogen en su seno a personas diversas de diferentes estatus socioculturales a quienes les une la necesidad de acercarse al conocimiento por puro placer, más que por una necesidad real.

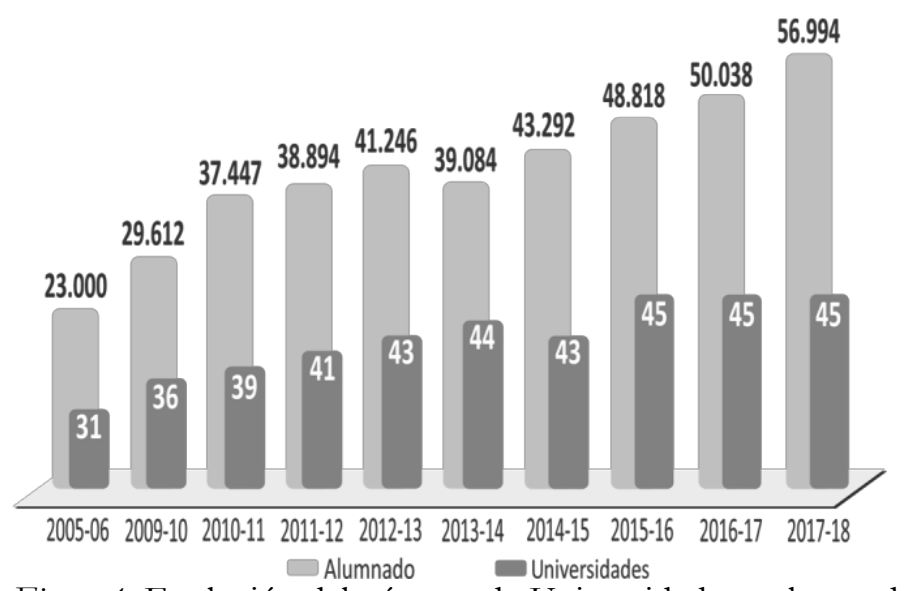

Figura 1. Evolución del número de Universidades y alumnado total matriculado.

Fuente: AEPUM(2018)

Por otra parte, siempre ha existido la preocupación de las universidades españolas por la extensión universitaria, la expansión de su conocimiento entre los distintos estratos sociales, pero en la actualidad resulta imprescindible que el conocimiento transcienda de los muros institucionales para mostrar la necesidad de generar conocimiento crítico y aplicado necesario para la transformación social. Que la práctica totalidad de las universidades españolas dediquen un espacio para este fin nos indica, además, de la responsabilidad social con la que se ejercen las tareas académicas, gestoras e investigadoras de nuestras universidades. Sin embargo, se precisa apostar por un modelo más integrador y transdisciplinar que permita abordar el conocimiento en su conjunto y supere alguna de las carencias actuales relacionadas con saberes parcelados que, siendo necesarios, dificultan el aprendizaje a largo de la vida desde una perspectiva crítica y emancipadora (Morín, Roger \& Domingo, 2013).

La oferta de PUM en las universidades españolas está generalizada, si bien nos encontramos dos tipos de programas universitarios para mayores. Por un lado, existen programas integrados en la vida académica y por otro lado programas específicamente ofertados a personas adultas diseñados para tal fin. En el primer caso, se trata de abrir las aulas universitarias convencionales de algunos 
Grados a las personas adultas con fines únicamente de desarrollo personal y cultural, es decir, son alumnos o alumnas de pleno derecho, pero sin reconocimiento académico alguno. Por ejemplo, la Universidad de Valladolid cuenta con la Universidad Permanente Millán Santos, que su modalidad abierta, consiste en ofertar a una persona adulta que se pueda matricular en alguna de las asignaturas convencionales de la Universidad que se proponen para tal fin. La persona mayor se convierte en alumno o alumna de la Universidad de Valladolid a todos los efectos y adquiere la obligación de asistir a clase con regularidad, pero sin derecho a evaluación ni a una acreditación de conocimiento adquirido.

Por otra parte, se imparten programas específicamente diseñados para personas mayores de 50, 55 o 60 años. Se trata de itinerarios formativos relacionados con diversos ámbitos disciplinares impartidos por profesorado universitario con una metodología específica que pretende acercar el mundo académico a estas personas. A esta segunda tipología corresponde el Programa Interuniversitario de la Experiencia de Castilla y León.

\section{El programa interuniversitario de la experiencia de Castilla y León}

El Programa Interuniversitario de la Experiencia (PIEX) un proyecto pionero en España que nace en el seno de la Universidad Pontificia de Salamanca en 1993 y rápidamente se va implantando en el resto de universidades a iniciativa de la Junta de Castilla y León. Actualmente, existen veintiocho sedes repartidas por tierras castellanoleonesas, en las que colaboran las ocho universidades, públicas y privadas, de la comunidad (Universidad de Burgos, Universidad de león, Universidad Pontificia de Salamanca, Universidad de Salamanca, Universidad de Valladolid, Universidad Europea Miguel de Cervantes, Universidad Santa Teresa de Jesús de Ávila y la IE University) y en el están matriculadas más de 6.000 personas.

El PIEX se enmarca en un programa integral de envejecimiento activo y del aprendizaje a lo largo de la vida, que pretende dar a las personas mayores la posibilidad de acceder a la cultura y la ciencia como fórmula de crecimiento personal, abriendo un espacio de intercambio generacional, y una vía de participación solidaria en la sociedad.

El programa, encuadrado en los itinerarios del aprendizaje a lo largo de toda la vida, aporta un conocimiento necesario para que los ciudadanos y ciudadanas puedan mejorar su calidad de vida y fomentar procesos de participación y socialización fuera de los espacios más habituales. Se trata de crear espacios en los que las personas decidan aprovechar su tiempo de forma activa, aprendan, disfruten de la cultura, creándola incluso, convirtiéndola en una plataforma para socializarse, y, en definitiva, seguir creciendo como ciudadanos y ciudadanas.

Este programa era una realidad exclusivamente urbana y desde el curso 2006-2007 empiezan a ponerse en marcha programas universitarios para personas adultas mayores en el mundo rural, con el objetivo de:

- Ofertar recursos sociales y educativos a los sectores rurales.

- Complementar la oferta de formación y participación social de los mayores de 55 años.

- Formar sobre temas que permitan mejor adaptación a los cambios sociales, culturales y tecnológicos.

- Integrar a los mayores en la comunidad universitaria.

En este contexto nace el Programa Interuniversitario de la Experiencia de Guardo, programa de la Junta de Castilla y León gestionado por la Universidad de Valladolid. Guardo es un pueblo situado al norte de la provincia de Palencia, a 98 kilómetros de la capital y que cuenta con más de 6.000 habitantes. Constituye el centro neurálgico de la zona nordeste de la provincia de Palencia, comarca caracterizada por la diversidad y la riqueza ecológica. Se trata del núcleo con mayor número de habitantes y uno de los de mayor tradición industrial y minera de Palencia, con una fuerte presencia 
actualmente del sector servicios. La localidad cuenta con un gran número de mineros prejubilados que demandan una cierta actividad cultural para evitar el traslado masivo de su residencia habitual a las zonas costeras. La proyección cultural del pueblo no es muy elevada y se centraba en la actividad que emana desde Ayuntamiento y Diputación basada en el acceso a las TIC, la prevención enfermedades y la actividad física o deportiva (Nieto \& Tejedor, 2006).

El programa Interuniversitario de la Experiencia de Guardo, al igual que en el resto de las sedes, es un programa cultural estructurado e impartido por profesorado universitario en el que se incluyen contenidos de literatura, medio ambiente, arte, cine, ciencia, interculturalidad, filosofía, antropología, psicología, sociología, educación para el desarrollo y la ciudadanía... En la actualidad, en las sedes rurales se ofrecen asignaturas de diversa índole agrupadas en torno a nueve itinerarios del conocimiento. Cada curso se seleccionan dos de ellos; en el curso 2018/19, se aborda un primer itinerario llamado "Cultura, Ciencia y Sociedad", que incluye asignaturas sobre ciencias de la vida, ciencias físico-químicas, política, sociología, economía, educación y sociedad, manifestaciones artísticas, comunicación. Y el segundo, "Geografía, Historia y Arte" en el que se ofertan materias de geografía (física, política, social, cartografía, climatología, espacios naturales y sociales), historia (etapas históricas, personajes y hechos históricos) y arte (arquitectura, artes plásticas, artes escénicas, escultura, pintura, música, cine, historia del arte).

Son muchas las mujeres y los varones que han habitado sus aulas durante más de veinte años. Son sus descripciones y valoraciones así como nuestras interpretaciones sobre su percepción del el Valor Social de la Educación las que fundamentan este trabajo.

Como se observa en la tabla 1, el número de asistentes ha ido aumentado en la sede rural de Guardo de forma paralela al descenso de participantes en la sede urbana de Palencia. Un argumento que puede ayudar a comprender este proceso está relacionado con la variedad de recursos culturales y educativos que existen en la ciudad, así como la diferencia cualitativa que supone una oferta como esta en el medio rural. Y es aquí donde queremos centrar el análisis del Valor Social que aporta la educación a lo largo de este trabajo.

Tabla 1.

Balance de matriculas del PIEX sedes Palencia y Guardo. Fuente: Coordinación PIEX

\begin{tabular}{|c|c|c|c|}
\hline Curso & $\begin{array}{c}\text { Matriculados en } \\
\text { Palencia }\end{array}$ & $\begin{array}{c}\text { Matriculados en } \\
\text { Guardo }\end{array}$ & Total \\
\hline $2004 / 2005$ & 121 & -- & 121 \\
\hline $2005 / 2006$ & -- & 25 & 25 \\
\hline $2006 / 2007$ & 109 & 36 & 145 \\
\hline $2007 / 2008$ & 117 & 51 & 168 \\
\hline $2008 / 2009$ & 130 & 52 & 182 \\
\hline $2009 / 2010$ & 116 & 48 & 164 \\
\hline $2010 / 2011$ & 125 & 53 & 178 \\
\hline $2011 / 2012$ & 114 & 58 & 172 \\
\hline $2012 / 2013$ & 91 & 53 & 144 \\
\hline $2013 / 2014$ & 99 & 52 & 151 \\
\hline $2014 / 2015$ & 93 & 59 & 152 \\
\hline $2015 / 2016$ & 93 & 53 & 146 \\
\hline $2016 / 2017$ & 98 & 62 & 160 \\
\hline $2017 / 2018$ & 91 & 61 & 152 \\
\hline
\end{tabular}

\section{Método de investigación}

El estudio realizado se centra en analizar el valor social que los y las participantes del PIEX de Guardo otorgan al programa (VSE Subjetivo), desde la reflexión de la importancia del mismo para sus vidas, a nivel cultural, personal y social. Se plantea como un estudio de caso que analiza el PIEX desde una doble perspectiva, personal y grupal, a través de dos procedimientos de recogida de 
información, entrevistas y cuestionarios, interpretando los mismos con la técnica de análisis del discurso que nos permite compartir el significado de la experiencia para el alumnado del programa.

Se han tomado como referencia las dimensiones e indicadores propuestos para el VSE objetivo y subjetivo indicados por Jornet, Perales y Sánchez-Delgado (2011) que se detallan en la siguiente tabla.

Tabla 2

Esquematización de las dimensiones de definición del constructo VSE. Fuente: Jornet, Perales y SánchezDelgado (2011, p.73-75).

\begin{tabular}{|c|c|c|}
\hline \multirow{9}{*}{$\begin{array}{l}\text { Valor Social } \\
\text { de la } \\
\text { Educación } \\
\text { (VSE) }\end{array}$} & \multirow{5}{*}{$\begin{array}{l}\text { Indicadores } \\
\text { sociales } \\
\text { VSE-Objetivo }\end{array}$} & D1. Dimensión y gasto en Educación \\
\hline & & D2. Familia y Educación \\
\hline & & $\begin{array}{l}\text { D3. Consecuencias educativas para el empleo a lo largo de la } \\
\text { vida }\end{array}$ \\
\hline & & $\begin{array}{l}\text { D4. Consecuencias sociales de tener ciudadanos bien } \\
\text { formados }\end{array}$ \\
\hline & & $\begin{array}{l}\text { D5. Educación como Política Social para promover la } \\
\text { Equidad y la Justicia Social }\end{array}$ \\
\hline & \multirow{4}{*}{$\begin{array}{l}\text { Percepciones } \\
\text { personales } \\
\text { VSE-Subjetivo }\end{array}$} & D1. Expectativas y metas educativas (alumnado y familia) \\
\hline & & D2. Justicia Social y Educación \\
\hline & & D3. Valor intrínseco diferencial de la Educación \\
\hline & & D4. Obstáculos y facilitadores del proceso educativo \\
\hline
\end{tabular}

De las dimensiones identificadas por Jornet, Perales y Sánchez Delgado (2011) para definir el constructo VSE-Subjetivo, la que mejor se ajusta a las características del PIEX como programa es la Dimensión 3 "valor intrínseco diferencial de la educación". Cuando las personas muestran el valor que le conceden al PIEX, como programa de formación adaptado a las personas adultas y mayores, que no va a tener un certificado que se pueda hacer valer en el mundo laboral, pero que sí parte de la curiosidad y las necesidades de este colectivo específico, están reconociendo "el valor intrínseco diferencial de la educación”, reflejando la importancia que conceden a la educación para la vida.

Dado el carácter especial del programa, no vinculado con la educación escolar, ni con el desarrollo laboral y profesional de las personas, ha sido necesario adecuar la definición del indicador, reemplazando lo "laboral" por lo "cultural".

Como procedimiento de recogida de información se ha utilizado, por un lado, un cuestionario diseñado para tal fin. En el mismo se pregunta a los y las participantes del PIEX de Guardo, a través de cuestiones abiertas, por las aportaciones fundamentales del programa en relación con conocimientos (cultural), desarrollo personal (personal) y convivencia e interrelaciones (social). En la última pregunta, se solicita que sinteticen en palabras clave su valoración general del programa. Para ello, se ofrece un listado de sustantivos, de entre los que se les pide que elijan cinco, dándoles además la opción de añadir abiertamente otras palabras que puedan ser más significativas. El cuestionario fue distribuido a todos los participantes de la sede de Guardo (61), recogiéndose un total de 38 cuestionarios (codificados como Sujeto 1 a Sujeto 38; S1 - S38); de ellos, 9 son hombres con una edad media de 70 años, 29 mujeres, con una edad media de 75 años.

Por otro lado, se han realizado cuatro entrevistas semi estructuradas a participantes paradigmáticos en lo que se refiere a edad, procedencia, nivel sociocultural y género (E1 a E4), todos del ámbito rural de Guardo.

Tanto las entrevistas como los cuestionarios se han analizado con técnica de análisis del discurso, tomando como referencia las categorías claves (cultural, social y personal) de las que se desprenden otras subcategorías y que constituyen el mapa fundamental, tal y como se presenta en la figura 2. 


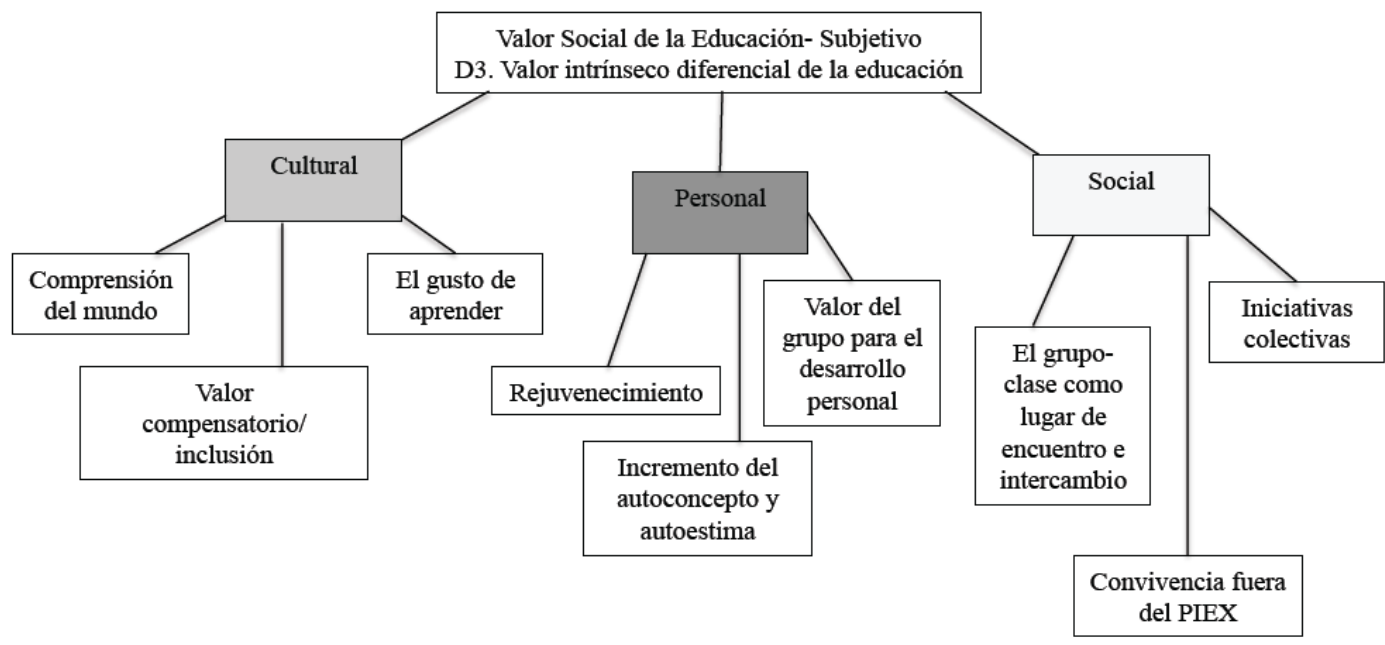

Figura 2. Categorías y subcategorías emergentes del discurso.

Fuente: elaboración propia.

\section{Las percepciones del alumnado en clave del valor social de la educación}

Son muchas y diversas las personas que han pasado por las aulas del PIEX, por lo que se ha considerado relevante recoger y analizar su discurso siguiendo las tres categorías señaladas con vistas a visibilizar el VS-subjetivo de la Educación que se desprende de esta experiencia.

En cuanto a la primera categoría, denominada "cultural", hace referencia al grado de adquisición de cultura, es decir, se ha analizado el conocimiento que se procesa, se genera y se comparte en este tipo de espacio. Entendiendo la necesidad de un constante reciclaje de aprendizajes y contenidos para entender la sociedad globalizada en la que vivimos, es interesante cómo el PIEX genera, por un lado, claves para una mejor comprensión del mundo actual, como señala una participante (E4) "aprender muchas cosas, por la variedad de temas, porque aporta cantidad de sentido a muchos aspectos culturales, sociales, históricos... que forman parte de nuestra vida cotidiana y nos ayuda a entenderla mejor". En el mismo sentido, otra persona (S26) señala "recordar, completar y actualizar conocimientos... una formación permanente para permanecer actualizada y que contribuye a completar mi formación integral como persona”. Y, por otro lado, ofrecer educación para todas las personas y colectivos, garantizando específicamente el acceso a la educación en las zonas rurales y la participación de las mujeres mayores de 55 años como sucede en la realidad que nos ocupa. Se trata, en este caso de una vivencia de aprendizaje a lo largo de la vida con una perspectiva compensatoria, son diversos los casos de mujeres que en su infancia o juventud no pudieron estudiar por la realidad social del momento, siendo en la actualidad cuando han podido ejercer ese derecho y manifiestan (S5, S12, S30) como lo aprendido cada día, no sólo sirve para mantener la mente activa, sino también para que se generen momentos de conversación con familiares y amigos favoreciendo así su inclusión y desarrollo.

Se trata de un proceso de aprendizaje estructurado en torno a itinerarios de asignaturas, organizado con ciertos márgenes de elección, lo que fomenta en el alumnado un mayor desarrollo, pues valoran que "las clases son amenas, adaptadas a todos los niveles del alumnado... y que no sean obligatorias y no haya exámenes también es un punto a favor, y en todo caso, lo que provoca en nosotros y nosotras es aún más interés y motivación" (E1). En la misma línea, también se destaca como el PIEX "da la posibilidad de aprender por el placer de aprender, sin necesidad de demostrar a nadie los conocimientos adquiridos" (S11). 
El profesorado, otra de las partes protagonistas del buen desarrollo del programa, es valorado por su implicación y capacidad de adaptar sus contenidos a la diversidad de niveles e intereses del alumnado. Los y las participantes (S7, S19, S21) valoran que no solo colabora de manera formativa, sino aportando también gran parte de su tiempo y sus conocimientos al servicio del aprendizaje a lo largo de la vida, compartiendo sus experiencias en los pasillos, cafetería, visitas culturales, tertulias, etc.

En segundo lugar, la categoría definida como "personal", resulta complicado extraer conclusiones que se puedan extrapolar a otros contextos, pero en este siglo en el que vivimos, donde la juventud es un valor siempre en alza y las industrias invierten millones en presentar lo joven como reclamo publicitario, es un verdadero placer escuchar a este hombre cuando dice que para él este programa ha sido como un renacer en su vida; volver a sentirse joven y vivo "me he dado cuenta de que he vuelto a ver amanecer en mi vida, tengo que reconocer que he rejuvenecido y me siento más joven" (E1).

Las realidades de las personas que asisten son múltiples, un aspecto que enriquece al programa, creándose también imaginarios compartidos, ya que muchos participantes señalan que el asistir a clase les hace sentirse bien consigo mismos y provoca un aumento de su autoestima. Para muchos su primer día de clase es vivido con nervios, inseguridades y miedo de hacer el ridículo (E2, E4, S1, S4, S5, S11, S13, S14, S18, S22, S23, S31, S33, S34), lo que provoca que en la primera sesión no salgan las palabras, pero después, como aporta una participante "poco a poco todo fluye y sientes que también puedes decir aportar" (E3). El PIEX provoca una rutina y un espacio de confianza que es vivido como un volver a empezar "estaba tranquila en casa, bueno, todo lo tranquila que se puede estar tras haber perdido quince días antes a mi marido, cuando una amiga me llamó para animarme a formar parte de esta experiencia, pese a mi pena interior, no quise perder la oportunidad que llevaba esperando toda la vida de poder asistir a clase y aprender muchas cosas. Participar en la Universidad de la Experiencia, me ha devuelto la alegría. He cambiado los antidepresivos por el aula, los compañeros, mejor dicho, los amigos y los profesores. Para mí ahora mismo todos ellos son mi familia" (E2). En la misma línea, otra participante señala "es como una terapia, porque he aprendido a comunicarme mejor, a ser más tolerante y ha mejorado mi autoestima, un poquito dañada desde el fallecimiento de mi marido" (E3).

El PIEX tiene un parón vacacional como en todos los programas educativos, pero vivido de forma totalmente contraria a como se vive en otras etapas vitales. Como señala una participante "eso que los jóvenes desean tanto, yo lo detesto. No me gustan las vacaciones, añoro todos los días la compañía que tengo durante el curso y el tiempo que dedico a aprender cosas nuevas e interesantes. Estoy todo el verano deseando que llegue octubre para comenzar un nuevo curso" (E2).

El PIEX pretende enseñar a comprender y disfrutar desde diferentes áreas de conocimientos (literatura, música, arte, cine, ciencia, tecnología, derecho, economía, teatro, genética, etc.), pero no asumiendo los contenidos indiscriminadamente, sino con una visión propia y crítica. Se contempla como una fuente de enriquecimiento personal y entenderlo como parte del patrimonio propio y ajeno. Año tras año, se suceden momentos repletos de aprendizajes y experiencias de sumo interés que confirman que el aprendizaje a lo largo de la vida es una realidad.

Por último, respecto a la categoría determinada como "social", se observa cómo el PIEX se presenta en Guardo como un espacio privilegiado de relaciones sociales que se transforman en una red de apoyos emocionales para las personas adultas y mayores de la localidad. Si bien casi todas y todos ellos son personas que ya se conocen entre sí, el hecho de coincidir en un espacio social y educativo diferente al habitual les permite presentarse y relacionarse de otra forma más enriquecedora, libre de prejuicios sobre asuntos profesionales o particulares vividos antaño. Algunos describen el PIEX como un regalo contra la inactividad, un espacio de convivencia y encuentro con otras personas. La convivencia y encuentro que se gestan en el espacio aula ha generado la búsqueda de otros espacios compartidos, como señala una de las participantes "me alegro mucho de tener tantos amigos dentro del grupo, somos todos como una piña y ya no nos conformamos con compartir dos horas de clase tres días a la semana, quedamos los fines de semana para cenar, hacemos 
excursiones nosotros mismos en las vacaciones, y nos juntamos muchos días para pasear o tomar un café. Somos una gran familia, que se va ampliando año a año con compañeros nuevos" (E4).

El PIEX se convierte en un espacio de relaciones, de encuentro, de amistades y favorece el bienestar de la persona y su función social una vez traspasada la frontera de la jubilación. Todo ello favorece seguir evolucionando como ciudadano y ciudadana desde una mirada de identidad de grupo donde todas las personas se reflejan en el otro, como señala uno de los participantes cuando habla de la idea de "camaradería" (S33). Se trata de lograr, a través de la educación, un desarrollo social que ayude a mejorar la vida personal y por ende la colectiva.

Es interesante cómo desde el programa se crean por parte de los participantes otros espacios de aprendizaje como son las "Tertulias", organizadas por el alumnado y en las que en ocasiones también participa el profesorado. Es, precisamente, en este espacio donde se respira claramente la idea de convivencia adulta; en ellas conviven personas de diferentes planteamientos ideológicos pero que se respetan y complementan con vistas a mejorar aprendizajes desde la colectividad sin perder la individualidad. Estas iniciativas propias, sumadas a la organización de jornadas y salidas culturales, han favorecido la creación de la Asociación de Alumnos y Antiguos Alumnos del PIEX de Guardo, tal y como sucede en otros programas de este tipo, que a su vez han ido conformado una amplia red de movimientos asociativos a nivel local, regional y nacional. De esta forma se han ido consolidando entidades como la Federación Regional de Castilla y León de Asociaciones de Alumnos y Antiguos Alumnos de la Universidad de la Experiencia (FASUNEX), la Asociación Española de Programas Universitarios para Mayores (AEPUM) y, la Confederación Estatal de Asociaciones y Federaciones de Alumnos y Ex alumnos de los Programas Universitarios de Mayores (CAUMAS).

En la última parte del cuestionario se recoge el significado que tiene el PIEX para los y las participante a través de palabras (ver figura 3). Es significativo desde los niveles de análisis que estamos manejando, observar como las palabras más repetidas son: amistad; compañerismo y cultura; aprendizaje y comunicación; reflexión; y participación, respeto y alegría.

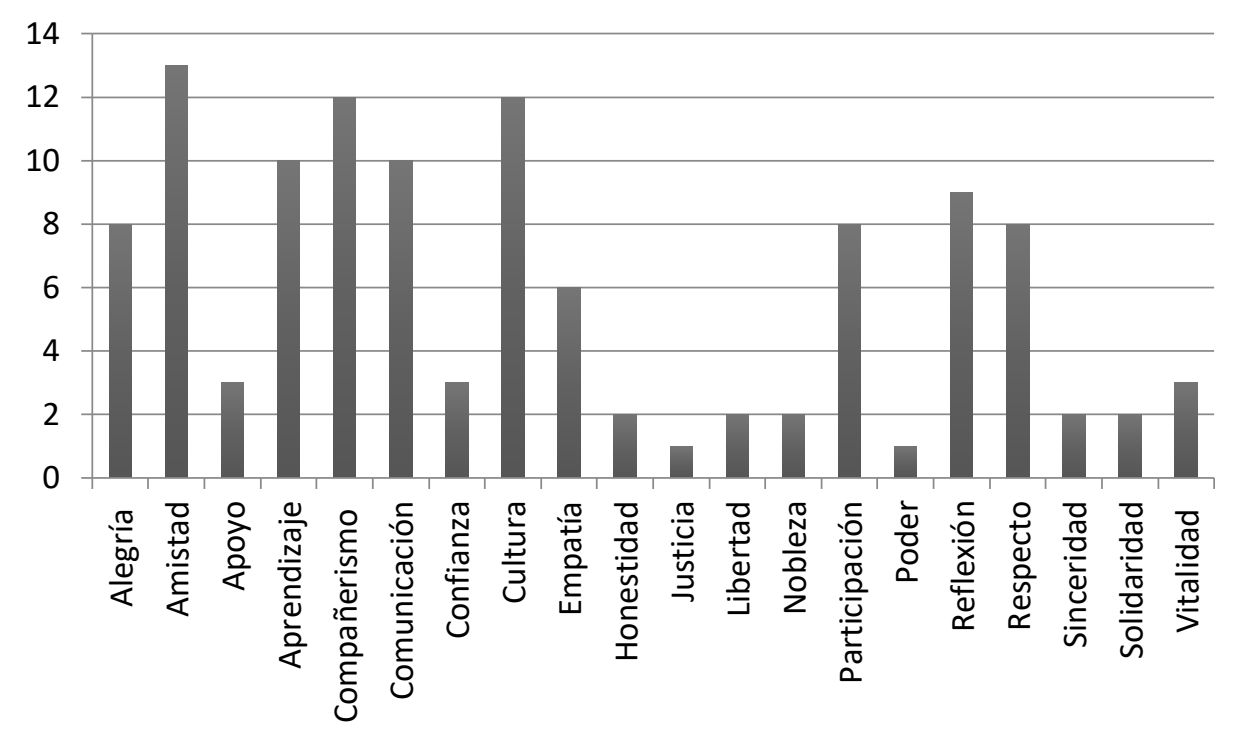

Figura 3. Palabras con las que identifican los y las participantes de Guardo (Palencia) el PIEX. Fuente: elaboración propia.

Las palabras seleccionadas por las personas participantes y sus significados están estrechamente relacionados con las dimensiones señaladas desde el objeto de estudio. Por un lado, desde un nivel cultural se observa el valor que dan a la cultura y al aprendizaje; desde un nivel personal el valor máximo a la amistad, seguida de compañerismo y alegría; y, en el nivel social, el valor que otorgan a la comunicación, reflexión, participación y respeto. Aspectos entrelazados que aportan una 
imagen significativa de lo que para ellos y ellas significa el PIEX en los tres planos de desarrollo contemplados (Figura 4).

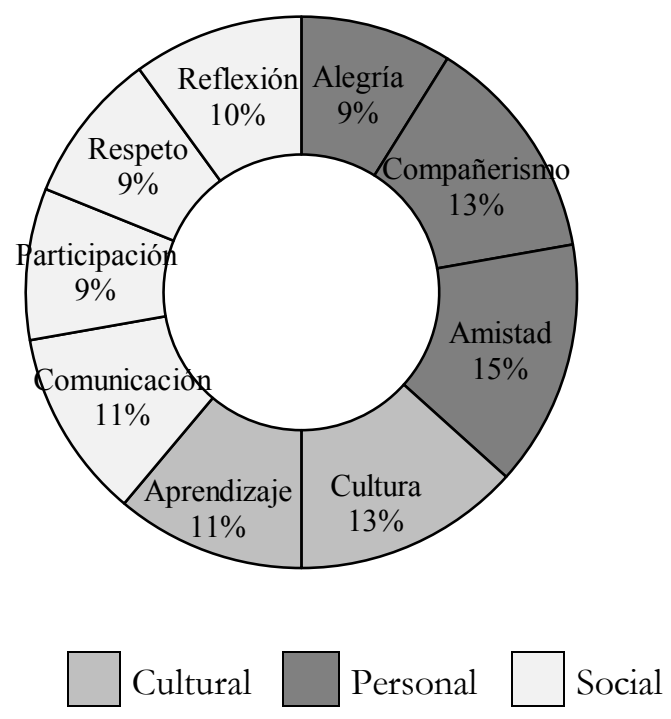

Figura 4. Palabras que identifican el PIEX a nivel cultural, personal y social.

Fuente: elaboración propia.

Todo lo señalado, junto con las afirmaciones de recomendación a otras personas para que participen en el PIEX, pone de manifiesto la valoración positiva del PIEX de Guardo (Palencia) por parte de sus participantes, convirtiéndose en un indicador del alto valor social concedido a la educación por este colectivo. Esto muestra la necesidad de señalar el valor social de los Programas Universitarios para Mayores y su orientación hacia el gusto por el aprendizaje, el conocimiento desinteresado, la cultura, la convivencia y el valor de la participación ciudadana. En este caso, además, asumiendo también una función preventiva en lo que se refiere a la enseñanza de pautas culturales que sean una alternativa a los ocios pasivos y que ofrezcan oportunidades sociales y educativas.

\section{Conclusiones}

En un mundo en constante transformación, la ciudadanía reclama espacios formativos, innovadores y de calidad, para poder ejercer eficazmente como miembros de un colectivo en el que el conocimiento y la preparación sean la base esencial desde la que afrontar los constantes procesos de cambio a los que están sometidos. Los Programas Universitarios para Mayores son escenarios donde se puede posibilitar la construcción de un pensamiento que proporcione a cada ser humano la capacidad de actuar en el contexto donde se ubique, convirtiéndose en espacios privilegiados de cultura y aprendizaje en los que se cultiva la educación para la convivencia y la participación, donde la educación social y ciudadana es un componente esencial.

Las diversas visiones del aprendizaje a lo largo de la vida y del futuro del mismo convergen hacia una misma idea: una educación integral, permanente, para todas las personas, sin distinciones de etnia, sexo, condición social... una educación que contribuya al desarrollo pleno tanto de la persona como de la sociedad, de forma que seamos capaces de convivir en un mundo en el que la paz, la justicia social y la cooperación, cobren el papel que realmente merecen, fomentando la solidaridad para luchar contra la exclusión.

La información, presentada a través de las valoraciones que hace el alumnado, es relevante y útil para seguir consolidando este tipo de programas que ponen la cultura al servicio de las personas mayores favoreciendo en el desarrollo cultural, personal y social. Todas ellas, en consonancia con el 
constructo que vertebra este trabajo, el Valor Social de la Educación subjetivo, cuya pretensión es corroborar que la educación se aproxima a la consecución de tales retos. No obstante, desde este trabajo, se considera imprescindible repensar las dimensiones del constructo cuando se refiere a acciones educativas enmarcadas en el ámbito de la educación social, favoreciéndose así posteriores análisis en clave de VSE en itinerarios educativos como el presentado. En esta ocasión, se ha transitado de lo escolar a lo social para adaptar la investigación ya realizada al contexto señalado, un aspecto que nos resulta de gran interés para seguir analizando el valor social de la educación en contextos sociales, teniendo como referencia otros programas y espacios.

Las aportaciones que hacen los participantes, además de enfatizar la valoración del programa, muestran un perfil de persona adulta o mayor caracterizado por valores vinculados a la participación, la vida activa y la motivación por aprender y compartir aprendizajes que influye directamente en el plano personal, cultural y social. En todos esos ámbitos incide la participación en el PIEX, perfilando nuevas formas de entender el envejecimiento y la función social de estas personas, concibiendo los distintos itinerarios educativos como espacios de convivencia y aprendizaje gracias al acceso a la cultura que influye en todos los planos de la vida de los participantes tanto a nivel individual como colectivo.

\section{Referencias bibliográficas}

Billet, S. (2002). Critiquing workplace learning discourses: participation and contiunity at work. Studies in Education of Adults, 34 (1), 56-67.

Cabalé, E. \& Rodríguez, G. R. (2017). Educación no formal: potencialidades y valor social. Revista Cubana de Educación Superior, 36 (1).

Cabello Martínez, M. J. (2002). Educación Permanente y Educación Social. Controversias y compromisos. Málaga: Aljibe.

Caride, J. A. (2004). No hay educación no formal. Educación Social. Revista de Intervención Socioeducativa, $28,6-9$.

Caride, J.A. (2004). ¿Qué espacios (y tiempos) de intervención socio-educativa en el mundo actual? Educar y educares en clave social: primeras respuestas a un interrogante. En I. P. Portugal (Ed.), IV Encontro Internacional Fórum do Paulo Freire. Oporto.

Coombs, P. H. (1971) La crisis mundial de la educación. Barcelona: Península.

Fernández-García, A.; García, J.L. \& Pérez, G. (2014). Los Programas Universitarios de Mayores y su contribución al aprendizaje a lo largo de la vida. Revista Complutense de Educación, 25 (2), 521-540.

Flecha, R., \& Elboj, C. (2000). La educación de personas adultas en la sociedad de la información. Educación XX1, 3, 141-162.

International Longevity Centre Brazil (2015). Envejecimiento Activo. Un marco politico ante la revolución de la longevidad. Rio de Janeiro: ILC-Brazil.

Jornet, J.M. (2012). Las Dimensiones Docentes y Cohesión Social: Reflexiones desde la Evaluación. Revista Iberoamericana de Evaluación Educativa, 5 (1), 350-362.

Jornet, J.M., Perales, M.J., \& Sánchez-Delgado, P. (2011). El valor social de la educación: entre la subjetivad y la objetividad. Consideraciones teórico-metodológicas para su evaluación. Revista Iberoamericana de Evaluación Educativa, 4 (1), 52-77.

Martí Puig, M., \& Gil Gómez, J. (2012). La falta de cursos de ignorancia. En M. Martí Puig, \& J. Gil Gómez, Aprendizaje Permanente. Competencias para una formación crítica: aprender a lo largo de la vida (1524). Xátiva: Edicions del Crec.

Moreno-Crespo, P. (2015). Educación a lo largo de la vida: Aulas de Mayores. Revista Fuentes, 17, 113 133.

Morin, E., Roger Cuirana, E. \& Domingo Mota, R. (2013) Educar en la era planetaria. Barcelona: Gedisa.

Morón Marchena, J.A. (2014). Educación y personas mayores. Revista Electrónica Interuniversitaria de Formación del Profesorado, 17 (1), 107-121. 
Nieto, M., \& Tejedor, M. (2006). Los programas universitarios para mayores del mundo rural: la experiencia de Guardo como un elemento de desarrollo para la mujer rural, en J.M. Esteve, Género y pedagogía social. Málaga: Universidad de Málaga.

Núñez, V. (2010). Trampas teóricas y conceptuales de la Pedagogía Social en construcción. Ponencia al Congreso Internacional de Pedagogia Social, Sao Paulo, Brasil.

Organización Mundial de la Salud-OMS (2002). Active Ageing: A Policy Framework. Ginebra: OMS.

Ortega, J. (1998). La educación a lo largo de la vida o el espacio de la educación social. En L. Pantoja, Nuevos espacios educativos en educación social. Bilbao: Mensajero.

Ortega, J. (2005). La Educación a lo Largo de la Vida: la educación social, la educación escolar, la educación continua... todas son educaciones formales. Revista de Educación, 338, 167-175.

Pozón, E. (2018). Asignatura pendiente en el siglo XXI. Conocer el proceso del envejecimiento y la etapa de la vejez. Libro Blanco de CAUMAS. Madrid: CAUMAS.

Quintano, J. (2014). Educación y participación ciudadana en la edad adulta y la vejez, en J.L. Hernández Huerta (coord.) (2014). En torno a la Educación Social. Estudios, reflexiones y experiencias (pp. 55-64). Salamanca: FahrenHouse.

Sancho-Álvarez, C., Jornet, J.M., \& Gonzáles-Such, J. (2016). El constructo Valor Social de la Educación; validación cruzada entre profesorado de escuela y universidad. Perfiles educativos, 34 (2), 329-350.

Sancho-Álvarez, C., Jornet, J.M., \& Gonzáles-Such, J. (2017). Una aproximación al valor subjetivo de la educación en Iberoamérica. Evidencias de validez en Argentina, España y república Dominicana. Revista de Investigación Educativa, 39 (157).

Tejedor, M., \& Rodríguez, H. (2008). Ciudadanía y aprendizaje dialógico en los programas universitarios para mayores: reflexiones y experiencias desde la pedagogía crítica. Educar, 42, $97-$ 115.

UNESCO. (2005). Hacia las sociedades del conocimiento. París:UNESCO.

UNESCO (2013). Declaración de Beijing sobre la Creación de Ciudades del Aprendizaje. Beijing: UNESCO

Vargas, C. (2017). El aprendizaje a lo largo de la vida desde una perspectiva de justicia social. Serie de documentos temáticos sobre Investigación y Prospectiva en Educación, 21. Paris, UNESCO.

Valle, C., \& Tejedor, M. (2006). Cultura, convivencia y aprendizaje diálogico en los programas universitarios para mayores. En Actas III Congreso de Programas Universitarios para Mayores. Almería: Universidad de Almería.

\section{Para citar este artículo}

Quintano Nieto, J., Ortega Gaite, S., \& Tejedor Mardomingo, M. (2018). Aportes de los Programas Universitarios para Mayores al Valor Social de la Educación a lo largo de la vida. Reflexiones a partir del estudio de un caso. Revista Fuentes, 20(2), 105-118. [Fecha de consulta: $\mathrm{dd} / \mathrm{mm} / \mathrm{aa}]$.

doi: http://dx.doi.org/10.12795/revistafuentes.2017.v20.i2.07 